\title{
New Insights into Guidance Laws with Terminal Angle Constraints
}

\author{
Chang-Hun Lee ${ }^{1}$ and Min-Guk Seo ${ }^{2}$ \\ Cranfield University, Cranfield, MK43 OAL, UK
}

\section{Introduction}

In the field of guidance technology, advanced guidance laws are being developed using various control theories such as optimal control [1-2], sliding mode control [3-6], H-infinity control [7], state-dependent Riccati equation (SDRE) [8], Lyapunov theory [9], geometric control theory [10], predictive control, [11-12], and feedback linearization control [13]. The general procedure of this approach is to first establish the guidance problem to be solved and to define the guidance geometry and kinematics equation corresponding to the guidance problem. A guidance law is then systematically designed in such a way that an appropriate control theory is applied to a predetermined guidance problem. The potential importance of this approach is that a number of advanced guidance laws can be newly developed depending on the combinations of guidance problems and control theories. Therefore, recent trends in the design of guidance law focus on finding a new combination of guidance problems and control theory to obtain a new guidance law that is superior to the conventional guidance law. While previous research has focused more on the methodology and the design process itself, little effort has been made towards understanding the characteristics of the newly developed guidance laws.

When implementing a newly developed guidance law in a real system, understanding and analyzing the nature of the guidance law is an important prerequisite for evaluating its reliability

\footnotetext{
${ }^{1}$ Research Fellow, School of Aerospace, Transportation, and Manufacturing, Cranfield University, Cranfield, MK43 0AL, United Kingdom/lckdgns@gmail.com (Corresponding Author)

${ }^{2}$ Research Fellow, School of Aerospace, Transportation, and Manufacturing, Cranfield University, Cranfield, MK43 0AL, United Kingdom
} 
and predicting its performance. In other words, in practical applications, we can apply the developed guidance law to a real system once it is judged that we have sufficient confidence in its properties. It is therefore necessary to at least understand how the guidance law will act in comparison with the existing guidance law. This can be done in a way that examines the physical meaning of the guidance law. However, since most of the advanced guidance laws developed in recent years are given in the form of complex guidance commands, it is difficult to grasp their physical meaning. Moreover, as mentioned above, efforts to understand this have not been undertaken so far.

Until recently, the optimal control theory has attracted considerable attention as a way to devise advanced guidance laws [14-25] due to its design methodology being well-structured and its ability to provide guidance laws in the form of state variable feedback. Also, it has been applied to many guidance problems regarding the interception of a target [14-17], as well as the impact angle control [18-25]. Particularly, in the impact angle control problems, optimal guidance laws using various types of cost functions [1-2, 19-25] have been devised. In the linearized kinematics, these guidance commands are given by a unified form with specific guidance gains $k_{1}$ and $k_{2}$.

$$
a_{M}=-\frac{k_{1}}{t_{g o}^{2}} y-\frac{k_{2}}{t_{g o}} v
$$

where $y$ and $v$ represent the lateral position and the lateral velocity in the linearized kinematics. The variable $t_{g o}$, called the time-to-go, represents the remaining intercept time. Recent studies have reported various sets of guidance gains depending on the choice of cost functions [1-2, 1925]. The unified form of the guidance command consists of two terms: feedback of lateral position and velocity weighted by the time-to-go. Since the optimal guidance command is 
obtained by simply finding a solution of the optimal guidance problem, the physical meaning of the derived guidance command is not explicitly shown. So even if the optimal guidance law seems to work, we cannot understand exactly how it works. This information is significant in implementing the guidance law.

Therefore, this paper focuses on understanding the physical meaning of each term of the optimal guidance command in order to facilitate readers' understanding. First, the original guidance gains are mapped to new guidance gains using the decomposition of values of the guidance gains. After mapping, the original guidance command composed of two terms can be converted to a new alternative guidance command for analysis purpose, in which the physical meaning of each term of the guidance command is explicitly displayed. Therefore, we can understand how the optimal guidance law works to satisfy the terminal constraints: it turns out the physical meaning of optimal guidance commands is the feedback of the heading angle error (proportional navigation guidance, PNG) and the feedback of the impact angle error. In this interpretation, the impact angle error is defined as the angle difference between the desired impact course and the predicted final flight path angle by PNG.

The governing equations of the heading angle error and impact angle error are derived, as well. We reveal the components of the actual guidance gains that contribute to reducing the heading angle error or impact angle error. Additionally, according to references $[2,19]$, the optimal guidance law with the impact angle constraint has been understood so far as the combination of PNG with the navigation constant $k_{2}$ and the additional bias term for the impact angle control. However, in this paper, we raise the problem that the existing interpretation of the optimal guidance laws needs to be corrected: the actual navigation constant is not $k_{2}$, and we provide the correct value. 
The presented results of this paper can be used to understand the characteristics of the optimal guidance laws and as a guideline for selecting appropriate guidance gains that reflect the physical meaning. Such an understanding can help increase the reliability and confidence of the optimal guidance laws when applying it to a real system.

This paper consists of four sections. In Section II, the problem this paper is seeking to solve is introduced. A new interpretation of optimal guidance law is given in Section III. Finally, we conclude our study in Section IV.

\section{Problem Formulation}

\section{A. Derivation of Engagement Kinematics}

We consider a planar engagement scenario for a stationary target shown in Fig. 1. Two types of coordinate systems called the inertial frame $\left(X_{I}-O-Y_{I}\right)$ and the reference frame $\left(X_{R}-O-Y_{R}\right)$ are introduced $[21,24]$. The reference frame is defined in the inertia frame to rotate by the desired impact angle $\gamma_{f}$. Missile and target positions are denoted by $M$ and $T$, respectively. The flight path angle and the line-of-sight (LOS) angle are indicated by $\gamma_{M}$ and $\sigma$, respectively. The two angles measured from the reference frame are denoted by $\bar{\gamma}_{M}$ and $\bar{\sigma}$, which are given as

$$
\bar{\gamma}_{M}=\gamma_{M}-\gamma_{f}, \quad \bar{\sigma}=\sigma-\gamma_{f}
$$

The other variables are self-explanatory. 


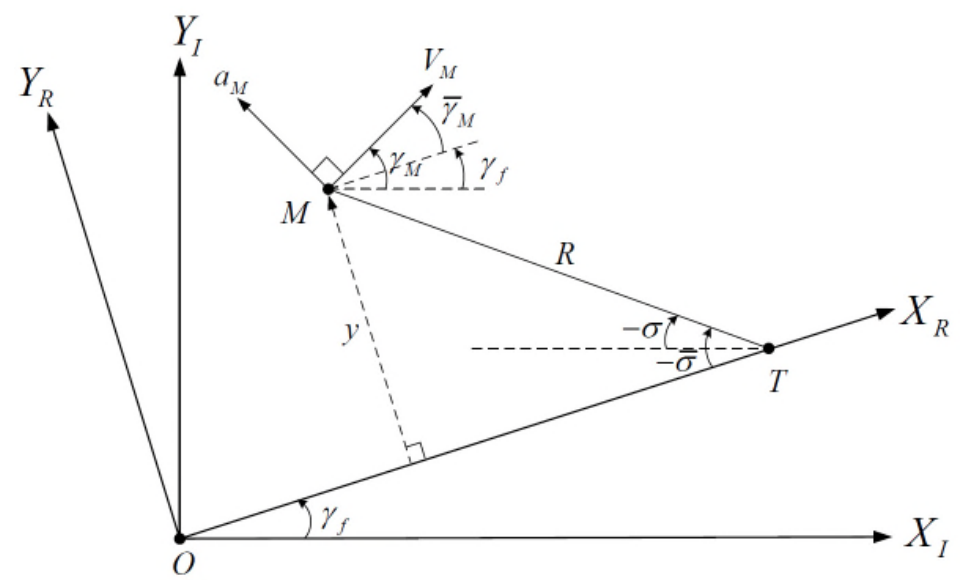

Fig. 1. The engagement geometry and parameter definitions

The nonlinear engagement kinematics in the reference frame can be expressed [21, 24].

$$
\begin{aligned}
& \dot{y}=V_{M} \sin \bar{\gamma}_{M} \\
& \dot{\bar{\gamma}}_{M}=a_{M} / V_{M}
\end{aligned}
$$

We assume that $V_{M}$ is constant and that the initial $\bar{\gamma}_{M}$ is small enough to be linearized by an appropriate mid-course guidance law. Then, the linearized kinematic kinematics can be obtained.

$$
\begin{aligned}
& \dot{y}=V_{M} \bar{\gamma}_{M}=v \\
& \dot{v}=a_{M}
\end{aligned}
$$

where $y$ and $v$ represent the position and velocity perpendicular to the $X_{R}$-axis, respectively. In the form of a matrix, the above equation can be expressed.

$$
\dot{x}=A x+B u, \quad x\left(t_{0}\right)=x_{0}
$$

where

$$
x \triangleq[y, v]^{T}, \quad u \triangleq a_{M}, \quad A \triangleq\left[\begin{array}{ll}
0 & 1 \\
0 & 0
\end{array}\right], \quad B \triangleq\left[\begin{array}{l}
0 \\
1
\end{array}\right]
$$

Remark 1: This linearized engagement kinematic is used for deriving optimal guidance commands [2, 16, 20-25]. And then, the final guidance commands for implementation are 
determined by replacing $y$ and $v$ with directly measurable parameters shown in Eq. (21) or Eq. (22).

\section{B. Derivation of Geometric Relationships}

Here we derive geometric relationships for later use. First, $\bar{\gamma}_{M}$ and $\bar{\sigma}$ can be expressed in terms of $y$ and $v$ from the engagement geometry shown in Fig. 1.

$$
\bar{\gamma}_{M}=v / V_{M}, \quad \bar{\sigma}=-y / R=-y /\left(V_{M} t_{g o}\right)
$$

In the above equation, $R$ is approximated by multiplying the missile velocity by time-to-go as $R \approx V_{M} t_{g o}$. Then, combining Eq. (2) and Eq. (7) yields.

$$
y=V_{M} t_{g o}\left(\gamma_{f}-\sigma\right), \quad v=V_{M}\left(\gamma_{M}-\gamma_{f}\right)
$$

Taking the time derivative of $y$ in Eq. (8) gives

$$
\dot{y}=-V_{M}\left(\gamma_{f}-\sigma\right)-V_{M} t_{g o} \dot{\sigma}=v
$$

Then, substituting Eq. (8) into Eq. (9) and rearranging yields

$$
\dot{\sigma}=-\left(y+v t_{g o}\right) /\left(V_{M} t_{g o}^{2}\right)
$$

It is well-known that the zero-effort-miss (ZEM) must be zero in order to intercept a target [19. In the linearized engagement kinematics, it can be expressed as the following.

$$
y+v t_{g o}=0
$$

Then, substituting Eq. (8) into Eq. (11) gives the following result.

$$
\sigma=\gamma_{M}
$$

Note that, for the stationary target, the condition of interception is to align the flight path angle with the LOS direction. In Eq. (8), it can be predicted that $v\left(t_{f}\right)$ must be zero for the satisfaction of the impact angle constraint. Also, in order to intercept a target, the missile must be placed on 
the collision line at the final time. Therefore, in the linearized kinematics, the conditions for satisfying the interception and desired impact angle are as follows.

$$
y\left(t_{f}\right)=v\left(t_{f}\right)=0
$$

\section{Class of Optimal Guidance Laws with Impact Angle Constraint}

The linear quadratic (LQ) optimal control problem for the impact angle control can be generalized as follows.

$$
\min _{u} J=\frac{1}{2} \int_{t_{0}}^{t_{f}} x^{T} Q x+R u^{2}(\tau) d \tau, \quad Q \geq 0, R>0
$$

with $x_{f}=[0,0]^{T}$. In the approach, the optimal guidance law is obtained by just solving Eqs. (14). The most interesting aspect of this approach is that the obtained optimal guidance law is expressed in the form of the state feedback as given in Eq. (1) with specific guidance gains. According to choices of the design parameters $Q$ and $R$, various sets of feasible guidance gains have been reported. In [19-20], an optimal guidance law (OGL) providing the desired impact angle was proposed. Its guidance gains are as follows

$$
U_{O G L}=\left\{k_{1}, k_{2} \in \mathbb{R}^{+} \mid k_{1}=6 \text { and } k_{2}=4\right\}
$$

By using the feedback of the impact angle error, an interception angle control guidance (IACG) law [21] has been devised. According to this study, the following set of guidance gains can also be used for the impact angle control.

$$
U_{\text {IACG }}=\left\{k_{1}, k_{2} \in \mathbb{R}^{+} \mid k_{1}=2\left(k_{2}-1\right) \text { and } k_{2} \geq 4\right\}
$$

In [22-23], the time-to-go weighted optimal guidance law (TWOGL) was devised and its guidance gains is given by

$$
U_{\text {TWOGL }}=\left\{k_{1}, k_{2} \in \mathbb{R}^{+} \mid k_{1}=\left(\left(k_{2}+1\right) / 2\right)^{2}-1 / 4 \text { and } k_{2} \geq 4\right\}
$$


The time-to-go polynomial guidance (TPG) law was also introduced in [24]. The set of guidance gains of TPG can be written as follows

$$
U_{T P G}=\left\{k_{1}, k_{2} \in \mathbb{R}^{+} \mid 2\left(k_{2}-1\right) \leq k_{1} \text { and } k_{1}<\left(\left(k_{2}+1\right) / 2\right)^{2} \text { and } k_{2}>3\right\}
$$

In addition, a generalized impact angle control guidance (GIACG) law for constant guidance gains has recently been developed in [25]. The guidance gains of GIACG are as follows.

$$
U_{\text {GIACG }}=\left\{k_{1}, k_{2} \in \mathbb{R}^{+} \mid 2\left(k_{2}-1\right) \leq k_{1} \text { and } k_{2}>3\right\}
$$

Fig. 2 shows all feasible sets of guidance gains for the class of the optimal guidance laws explained above. From this figure, we can readily observe the following relation of inclusion.

$$
U_{O G L} \subset\left(U_{I A C G}, U_{T W O G L}\right) \subset U_{T P G} \subset U_{G I A C G}
$$

Remark 2: If the guidance gains $k_{1}$ and $k_{2}$ are selected in the above sets, then the guidance command shown in Eq. (1) becomes optimal commands that minimize certain cost functions, depending on $Q$ and $R$ in Eq. (14). The information on the relationship between the guidance gains (i.e., $k_{1}$ and $k_{2}$ ) and $Q$ and $R$ in the objective function can be found in reference [25].

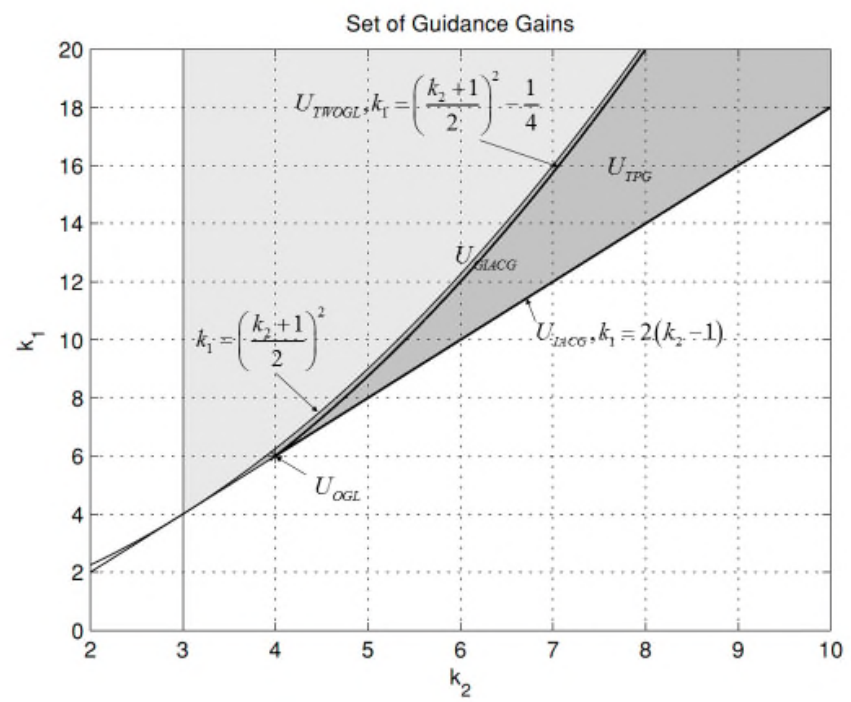

Fig. 2. Feasible sets of guidance gains 


\section{Problem Statements}

This section raises the main problem addressed in this paper. In order to implement the optimal guidance laws, the linearized form shown in Eq. (1) is not desirable, so the linearized state variables should be replaced with the original nonlinear and measurable parameters. Accordingly, by substituting Eq. (8) into Eq. (1), an alternative form of guidance command for implementation is obtained as

$$
a_{M}=\left(V_{M} / t_{g o}\right)\left[k_{1} \sigma-k_{2} V_{M}+\left(k_{2}-k_{1}\right) \gamma_{f}\right]
$$

Also, substituting Eqs. (10) and (8) into Eq. (1) gives a different implementation form of guidance command as

$$
a_{M}=k_{2} V_{M} \dot{\sigma}+\left(k_{2}-k_{1}\right) V_{M}\left(\gamma_{f}-\sigma\right) / t_{g o}
$$

When we apply these command forms to a nonlinear engagement scenario, we can see that these guidance commands work. However, it is difficult to see how they work physically. Additionally, it is not explicitly shown how the heading angle error and impact angle error behave as well as how the guidance gains are related to reducing those errors. Therefore, we want to bring up the following questions.

Q1. What is the actual physical meaning of the optimal guidance commands?

Q2. What are the governing equations for the heading angle error and impact angle error? Which parameters are related to the governing equations?

Additionally, according to reference [2, 19], based on the form of the guidance command shown in Eq. (22), the optimal guidance laws have been interpreted as PNG with an effective navigation constant $k_{2}$ plus an extra term. Thus, it has been accepted as fact so far that the optimal guidance laws become PNG with an effective navigation constant $k_{2}$ as the missile 
approaches a target. In this paper, we additionally raise the problem that the existing interpretation, which has been accepted as fact so far, needs to be revised.

\section{Interpretation of Optimal Guidance Laws}

This section deals with a new interpretation of the optimal guidance laws in order to respond the questions raised in the previous section. First, a new alternative form of the optimal guidance laws is derived for analysis purpose by using the decomposition of values of the guidance gains and rearranging the guidance commands. Following that, an effort is made to newly understand the characteristics of the optimal guidance laws by analyzing this form.

\section{A. New Alternative Form of Optimal Guidance Law}

First, it is assumed that the original guidance gains $k_{1}$ and $k_{2}$ that satisfy the conditions in Section II. C can be decomposed into new guidance gains $N$ and $\alpha$ as follows.

$$
k_{1}=N(\alpha+1), \quad k_{2}=N+\alpha, \quad \text { where } N, \alpha \in \mathbb{R}^{+}
$$

The above equation can be regarded as a nonlinear mapping function as $f:(N, \alpha) \rightarrow\left(k_{1}, k_{2}\right)$. Then, by substituting Eq. (23) into Eq. (1) and rearranging the result, the guidance commands can be rewritten as

$$
a_{M}=-N\left(\frac{y+v t_{g o}}{t_{g o}^{2}}\right)-\frac{N \alpha}{t_{g o}^{2}} y-\frac{\alpha}{t_{g o}} v
$$

Substituting Eq. (10) into Eq. (24) gives the following result.

$$
a_{M}=a_{M, P N G}+a_{M, B}
$$

where

$$
a_{M, P N G}=N V_{M} \dot{\sigma}, \quad a_{M, B}=-\frac{N \alpha}{t_{g o}^{2}} y-\frac{\alpha}{t_{g o}} v
$$


Note that the first term of Eq. (25) is identical to PNG with the navigation constant $N$. In order to analyze the physical meaning of PNG command, we rewrite the term associated with PNG command. By using Eqs. (8) and (10), the LOS rate is rewritten as $\dot{\sigma}=\varepsilon_{h} / t_{g o}$ where $\varepsilon_{h} \triangleq \sigma-\gamma_{M}$. Substituting this expression into $a_{M, P N G}$ gives

$$
a_{M, P N G}=\left(N V_{M} / t_{g o}\right) \varepsilon_{h}
$$

From Eq. (27), $\varepsilon_{h}$ can defined as the heading angle error for a stationary target. Therefore, the physical meaning of this term can be regarded as the feedback control command on the heading angle error with a time-varying proportional gain (P-gain). The role of this term is to make $\gamma_{M}=\sigma$

Next, we investigate the physical meaning of $a_{M, B}$. Under the condition of $a_{M, B}=0$, the optimal guidance commands become PNG as $a_{M}=a_{M, P N G}$. In this case, applying the kinematic relationship as $V_{M} \dot{\gamma}_{M}=a_{M}$ to the expression of $a_{M, P N G}$ gives $\dot{\gamma}_{M}=N \dot{\sigma}$. Then, integrating this term yields

$$
\gamma_{M_{f}}-\gamma_{M}=N\left(\sigma_{f}-\sigma\right)
$$

where $\gamma_{M_{f}}$ and $\sigma_{f}$ represent $\gamma_{M}\left(t_{f}\right)$ and $\sigma\left(t_{f}\right)$ respectively. From the condition as shown in Eq. (12), $\sigma_{f}=\gamma_{M_{f}}$ to intercept the target. Substituting this condition to Eq. (28) gives

$$
\gamma_{M_{f}}=\frac{N}{N-1} \sigma-\frac{1}{N-1} \gamma_{M}
$$

Note that the above equation can be regarded as an estimate of the flight path angle obtained at the final time when PNG with the navigation constant $N$ is applied. Next, substituting Eq. (8) into the expression of $a_{M, B}$ and rearranging the result yields 


$$
a_{M, B}=-\alpha(N-1) \frac{V_{M}}{t_{g o}} \gamma_{f}+\alpha(N-1) \frac{V_{M}}{t_{g o}}\left(\frac{N}{N-1} \sigma-\frac{1}{N-1} \gamma_{M}\right)
$$

By using the expression shown in Eq. (29), the above equation can be rewritten as follows.

$$
a_{M, B}=-\left[\alpha(N-1) V_{M} / t_{g o}\right] \varepsilon_{\gamma}
$$

where $\varepsilon_{\gamma} \triangleq \gamma_{f}-\gamma_{M_{f}}$. Note that this command is similar to $a_{M, P N G}$ as shown in Eq. (27). In the above equation, $\varepsilon_{\gamma}$ can defined as the impact angle error, which is the angle difference between the target impact angle and the estimate of the flight path angle achieved at the final time when PNG with the navigation constant $N$ is applied. Thus, the physical meaning of $a_{M, B}$ can be regarded as the feedback control command on the impact angle error based on the predictorcorrector approach. The term $-\alpha(N-1) V_{M} / t_{g o}$ can be regarded as a time-varying P-gain of the impact angle error. The role of this term is to make $\gamma_{M_{f}}=\gamma_{f}$. This term disappears when it is judged that the target impact angle can be satisfied by PNG command alone. In that case, the optimal guidance laws are automatically converted to PNG with the navigation constant $N$. Accordingly, unlike the previous understanding $[2,19]$, we can readily observe that the optimal guidance laws will have similar characteristics of PNG with the navigation constant $N=k_{2}-\alpha$, rather than $k_{2}$.

Finally, by combining Eqs. (27) and (31), the optimal guidance laws can be written

$$
a_{M}=N \frac{V_{M}}{t_{g o}} \varepsilon_{h}-\alpha(N-1) \frac{V_{M}}{t_{g o}} \varepsilon_{\gamma}
$$

with specific $N$ and $\alpha$ which are given in Eq. (23). It can be interpreted as a linear combination of the feedback control commands on the heading angle error and impact angle error. Fig. 3 shows the block diagram representation for working principle of optimal guidance laws. The 
impact angle control problem is defined as a kind of under-actuation control problem: controlling two independent variables (i.e., the heading angle error and the impact angle error) simultaneously by one control input (acceleration). This is the reason why the optimal guidance commands are given by the linear combination with specific feedback gains $N$ and $-\alpha(N-1)$.

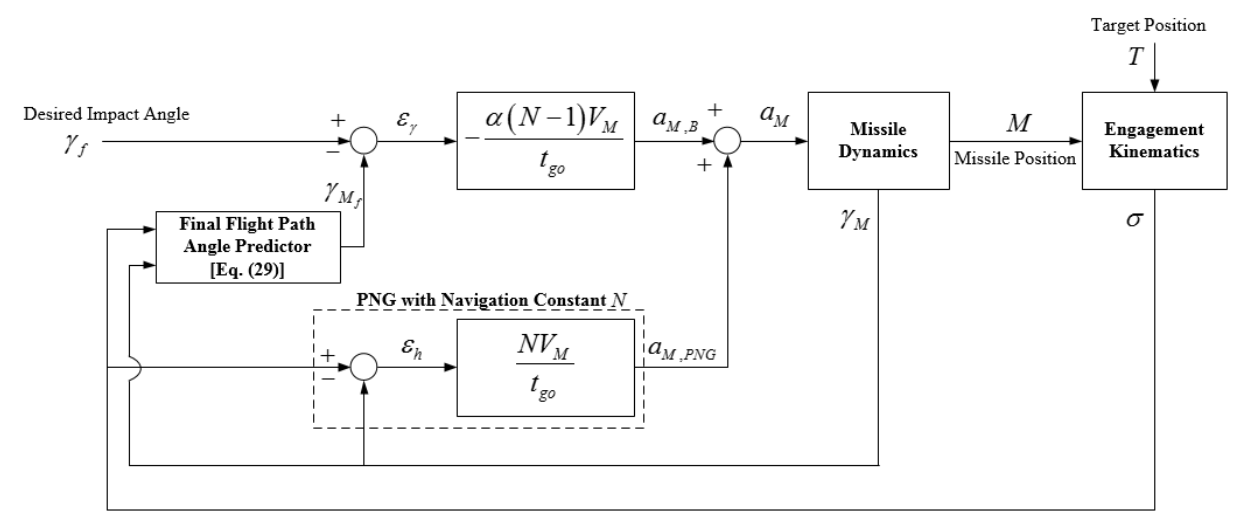

Fig. 3. Block diagram representation for working principle of optimal guidance laws

\section{B. Governing Equation for Heading Angle Error and Impact Angle Error}

First, we derive the governing equation of the impact angle error. From Eq. (29), taking the time derivative of $\dot{\gamma}_{M_{f}}$ and substituting $\dot{\sigma}=\varepsilon_{h} / t_{g o}$ into the result gives

$$
\dot{\gamma}_{M_{f}}=\frac{N}{N-1} \frac{\varepsilon_{h}}{t_{g o}}-\frac{1}{N-1} \dot{\gamma}_{M}
$$

From the kinematics relationship as $\dot{\gamma}_{M}=a_{M} / V_{M}$ and Eq. (32), we have

$$
\dot{\gamma}_{M}=N \frac{\varepsilon_{h}}{t_{g o}}-\alpha(N-1) \frac{1}{t_{g o}} \varepsilon_{\gamma}
$$

Then, substituting Eq. (34) into Eq. (33) yields

$$
\dot{\gamma}_{M_{f}}=\frac{\alpha}{t_{g o}} \varepsilon_{\gamma}
$$

Taking time derivative of $\varepsilon_{\gamma}$ gives 


$$
\dot{\varepsilon}_{\gamma}=\dot{\gamma}_{f}-\dot{\gamma}_{M_{f}}=-\dot{\gamma}_{M_{f}}
$$

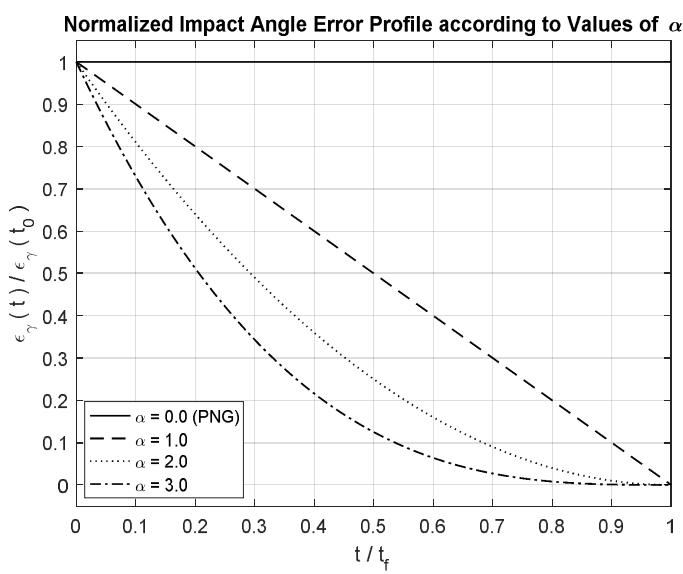

Fig. 4. Normalized impact angle error profiles for various $\alpha$

By substituting Eq. (35) into Eq. (36), we have

$$
\dot{\varepsilon}_{\gamma}+\frac{\alpha}{t_{g o}} \varepsilon_{\gamma}=0
$$

This equation can be seen as the governing equation of the impact angle error. Note that it is influenced by $\alpha$ only. Since Eq. (37) is a Cauchy-Euler equation, the closed form solution of (37) can be obtained by assuming $\varepsilon_{\gamma}(t)=t_{g o}^{\lambda}$ where $\lambda$ is some constant, as follows

$$
\varepsilon_{\gamma}(t)=C_{1} t_{g o}^{\alpha}
$$

where $C_{1}=\varepsilon_{\gamma}\left(t_{0}\right) / t_{f}^{\alpha}$. As shown in Eq. (38), for $\alpha>0$, it is clear that $\varepsilon_{\gamma} \rightarrow 0$ as $t_{g o} \rightarrow 0$. The value $\alpha$ determines the convergence speed of the impact angle error. Fig. 4 shows the normalized impact angle error profiles by $\varepsilon_{\gamma}\left(t_{0}\right)$ for various $\alpha$. As shown in Fig. 4 , as the value $\alpha$ increases, the convergence speed of the impact angle error also increases.

Next, the governing equation regarding the heading angle error will be obtained. Taking time derivative $\varepsilon_{h}$ yields

$$
\dot{\varepsilon}_{h}=\dot{\sigma}-\dot{\gamma}_{M}
$$


By combining Eqs. (8), (10), and (32) we have

$$
t_{g o} \dot{\varepsilon}_{h}+(N+\alpha-1) \varepsilon_{h}-\alpha(N-1)\left(\gamma_{f}-\sigma\right)=0
$$

Taking time derivative of Eq. (40) and rearranging the result with respect to $\varepsilon_{h}$ gives the following result.

$$
\ddot{\varepsilon}_{h}+\frac{(N+\alpha-2)}{t_{g o}} \dot{\varepsilon}_{h}+\frac{\alpha(N-1)}{t_{g o}^{2}} \varepsilon_{h}=0
$$

It can be regarded as the governing equation of the heading angle error. It is also expressed by a Cauchy-Euler equation. Accordingly, in the similar way, the closed form solution of $\varepsilon_{h}$ is obtained as follows.

$$
\varepsilon_{h}(t)=C_{2} t_{g o}^{N-1}+C_{3} t_{g o}^{\alpha}
$$

where $C_{2}$ and $C_{3}$ are constants determined by the initial values $\varepsilon_{h}\left(t_{0}\right)$ and $\dot{\varepsilon}_{h}\left(t_{0}\right)$, and the interception time, $t_{f}$ as follows.

$$
C_{2}=-\frac{\left[\alpha \varepsilon_{h}\left(t_{0}\right)+t_{f} \dot{\varepsilon}_{h}\left(t_{0}\right)\right]}{(N-\alpha-1) t_{f}^{N-1}}, \quad C_{3}=\frac{\left[(N-1) \varepsilon_{h}\left(t_{0}\right)+t_{f} \dot{\varepsilon}_{h}\left(t_{0}\right)\right]}{(N-\alpha-1) t_{f}^{\alpha}}, \text { where } N \neq \alpha+1
$$

As shown in Eq. (42), note that $\varepsilon_{h} \rightarrow 0$ as $t_{g o} \rightarrow 0$ under the conditions $N>1$ and $\alpha>0$. Also, the convergence pattern of the heading angle error depends on the choice of $N$ and $\alpha$.

In the case of PNG with the navigation constant $N$, the closed form solution of the heading angle error is given by

$$
\varepsilon_{h}(t)=C_{2}^{\prime} t_{g o}^{N-1}
$$

where $C_{2}^{\prime}=\varepsilon_{h}\left(t_{0}\right) / t_{f}^{N-1}$. Note that unlike PNG, there is the additional term in the optimal guidance laws as shown in Eq. (42). This term produces a different convergence pattern of $\varepsilon_{h}$ 
compared to PNG. This pattern is affected by the sign of $C_{2}$ and $C_{3}$. Also, the initial homing conditions $\varepsilon_{h}\left(t_{0}\right)$ and $\dot{\varepsilon}_{h}\left(t_{0}\right)$ determine the sign of $C_{2}$ and $C_{3}$, as shown in Eq. (43). When there is the same sign, this term can increase the convergence speed compared to PNG. If there are different signs, this term acts as a resistance of the convergence speed. In the physical sense, it indicates that for some initial homing conditions, an initial maneuver deviating from the collision course (increasing heading angle error) is required in order to achieve the desired impact course. Also, the convergence pattern of the heading angle error depends on the choice of $N$ and $\alpha$. As an example, Fig. 5 shows the normalized heading angle error profiles by $\varepsilon_{h}\left(t_{0}\right)$ for various $N$ and $\alpha$ under the condition $\dot{\varepsilon}_{h}\left(t_{0}\right)=0$.

The above equations as shown in Eqs. (38) and (42) can predict how the heading angle error and impact angle error converge. Therefore, the results can be used as a guideline for selecting the guidance gain related to these errors.

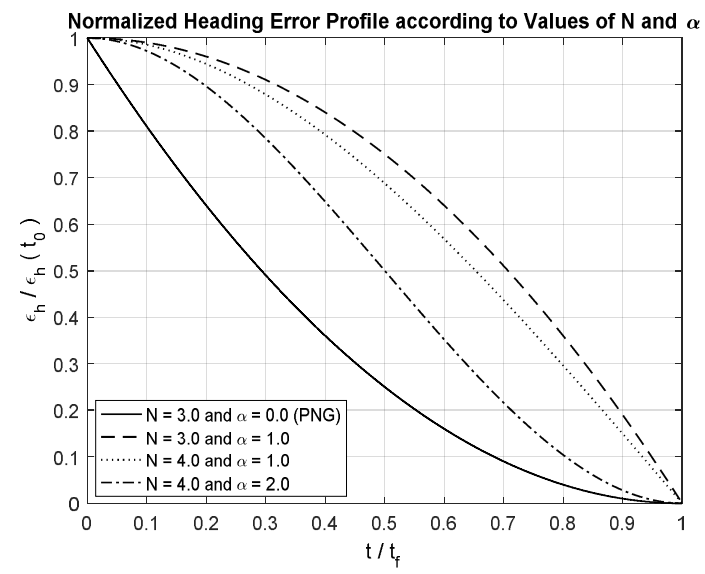

Fig. 5. Normalized heading angle error profiles for various $N$ and $\alpha$

Remark 3: For given guidance gains $k_{1}$ and $k_{2}$, there is only one solution of $N$ and $\alpha$ from Eq. (23) if $\left(k_{2}+1\right)^{2} / 4=k_{1}$. Under the condition of $\left(k_{2}+1\right)^{2} / 4>k_{1}$, there are two admissible solutions of $N$ and $\alpha$ : the first solution satisfies the condition $N>\alpha+1$ and the second 
solution satisfies the condition $N<\alpha+1$. That is because the nature of the impact angle control guidance laws: some of the guidance commands to reduce the impact angle error can also help reduce the heading angle error. Thus, a portion of the guidance command for the impact angle error can be expressed by a guidance command for the heading angle error, vice versa. Therefore, a single guidance command can be expressed in two ways. In other words, for a guidance command with the guidance gains $k_{1}$ and $k_{2}$, two interpretations (with different combinations of $N$ and $\alpha$ ) are possible. In both solutions, the flight trajectory, the pattern of flight path angle, and the convergence pattern of the heading angle error are the same, however the definitions of the impact angle error are different each other. Accordingly, the convergence patterns (or governing equations) of the corresponding impact angle error are also differently given.

\section{New Sets of Guidance Gains}

In the previous section, we have found the actual portions of the guidance gains $N$ and $\alpha$ which are directly related to the governing equations of the heading angle error and impact angle error in the original guidance gains $k_{1}$ and $k_{2}$. Namely, using the new guidance gains instead of the original guidance gains makes it more intuitive to predict the guidance performance as the guidance gains change. Therefore, this section will determine the feasible gain sets represented by $N$ and $\alpha$ from the original gain sets shown in Fig. 2. As shown in Eq. (20), $U_{\text {GIACG }}$ is the largest feasible gain set. Therefore, all feasible conditions of $N$ and $\alpha$ can be determined using the conditions of $U_{\text {GIACG }}$ shown in Eq. (19). Substituting Eq. (23) into the conditions of Eq. (19) gives

$$
(N-2)(\alpha-1) \geq 0 \quad \text { and } \quad N+\alpha>3
$$

Also, from Eq. (43), we have

$$
N \neq \alpha+1
$$


Therefore, any positive values of $N$ and $\alpha$ that satisfy Eqs. (45) and (46) simultaneously can be available for new guidance gains.

\section{Conclusions}

This paper aims to analyze the actual physical meaning of the optimal guidance laws with the impact angle constraint in order to facilitate readers' understanding. It is important to understand the characteristics of guidance laws implemented from a physical point of view in order to have confidence in the performance and reliability of the guidance laws in real applications. By decomposing the original guidance gains of the optimal guidance laws $\left(k_{1}\right.$ and $\left.k_{2}\right)$ with new guidance gains ( $N$ and $\alpha$ ), we derive new alternative form of the optimal guidance commands using them for analysis purpose. In this form, the physical meaning of the optimal guidance commands is explicitly presented. The optimal guidance commands can be interpreted as a linear combination of the feedback control commands on the heading angle error and impact angle error. In our interpretation, the definition of the heading angle error is the same as the existing definition. The impact angle error is defined as the angle difference between the target impact angle and the expected final flight path angle in PNG with the navigation constant $N$. Through analysis, we also show that unlike the existing interpretation that is accepted as fact so far, the optimal guidance laws behave with similar characteristics of PNG with the navigation constant $N<k_{2}$ (not $k_{2}$ ) as the missile approaches a target. In addition, the governing equations of the impact angle error and the heading angle error are derived. The first one is given by the firstorder differential equation and the closed form solution is given by a time-to-go polynomial function with an order of $\alpha$. The second one is expressed by the second-order differential equation and the closed form solution is given by a time-to-go polynomial functions with orders of $N-1$ and $\alpha$. This analysis reveals the components of the guidance gains that are directly 
related to the heading angle control and impact angle control, which are not clearly shown in the original guidance gains. The presented result in this paper not only helps to understand the characteristics of the optimal guidance laws but also can be used as a guideline for selecting the guidance gains that reflect the physical meaning.

\section{References}

[1] A. E. Bryson Jr. and Y.-C. Ho, Applied Optimal Control, New York: Wiley, 1975, pp. 154155.

[2] J. Z. Ben-Asher and I. Yaesh, Advances in Missile Guidance Theory, Vol. 180, Reston: AIAA, 1988, pp. 22-35.

[3] Moon, J., and Kim, Y., "Design of Missile Guidance Law via Variable Structure Control," Journal of Guidance, Control, and Dynamics, Vol. 24, No. 6, 2001, pp. 659-664, DOI: $10.2514 / 2.4792$

[4] S. D. Brierly and R. Longchamp, "Application of Sliding Mode Control to Air-Air Interception Problem," IEEE Transactions on Aerospace and Electronic Systems, Vol. 26, No. 2, 1990, pp. 306-325, DOI: 10.1109/7.53460

[5] A. Koren, M. Idan, G. M. Golan, "Integrated Sliding Mode Guidance and Control for Missile with On-Off Actuators," Journal of Guidance, Control, and Dynamics, Vol. 31, No. 1, 2008, pp. 204-214, DOI: $10.2514 / 1.31328$

[6] Y. B. Shtessel and C. Tournes, "Integrated Higher-Order Sliding Mode Guidance and Autopilot for Dual Control Missiles," Journal of Guidance, Control, and Dynamics, Vol. 32, No.1, 2009, pp. 79-94, DOI: 10.2514/1.36961 
[7] C. D. Yang and H. Y. Chen, "Nonlinear $H_{\infty}$ Robust Guidance Law for Homing Missiles," Journal of Guidance, Control, and Dynamics, Vol. 21, No. 6, 1998, pp. 882-890, DOI: $10.2514 / 2.4321$

[8] A. Ratnoo and D. Ghose, "State-Dependent Riccati-Equation-Based Guidance Law for Impact-Angle-Constrained Trajectories”, Journal of Guidance, Control, and Dynamics, Vol. 32, No. 1, 2009, pp. 320-326, DOI: 10.2514/1.37876

[9] A. Saleem and A. Ratnoo, "Lyapunov-Based Guidance Law for Impact Time Control and Simultaneous Arrival", Journal of Guidance, Control, and Dynamics, Vol. 39, No. 1, 2016, pp. 164-173, DOI: 10.2514/1.G001349

[10] S. Bezick, I. Rusnak, and W. S. Gray, "Guidance of a Homing Missile via Nonlinear Geometric Control Method," Journal of Guidance, Control, and Dynamics, Vol. 18, No. 3, 1995, pp. 441-448, DOI: $10.2514 / 3.21407$

[11] S. E. Talole, and R. N. Banavar, "Proportional navigation through predictive control," Journal of Guidance, Control, and Dynamics, Vol. 21, No. 6, 1998, pp. 1004-1006, DOI: $10.2514 / 2.4339$

[12] H. B. Oza and R. Padhi, "Impact-Angle-Constrained Suboptimal Model Predictive Static Programming Guidance of Air-to-Ground Missiles," Journal of Guidance, Control, and Dynamics, Vol. 35, No. 1, 2012, pp. 153-164, DOI: 10.2514/1.53647

[13] P. K. Menon, G. D. Sweriduk, and E. J. Ohlmeyer, "Optimal Fixed-Interval Integrated Guidance-Control Law for Hit-to-Kill Missiles," AIAA Guidance, Navigation, and Control Conference and Exhibit, Austin, Texas, 2003, DOI: 10.2514/6.2003-5579 
[14] Rusnak, I., "Optimal Guidance Law with Uncertain Time-of-Flight,” IEEE Transactions on Aerospace and Electronic Systems, Vol. 36, No. 2, 2000, pp. 721-725, DOI: $10.1109 / 7.845272$

[15] I. Rusnak, "Guidance Law Based on an Exponential Criterion for Acceleration Constrained Missile and a Maneuvering Target," Journal of Guidance, Control, and Dynamics, Vol. 19, No. 3, 1996, pp.718-721, DOI: 10.2514/3.21684

[16] J. Z. Ben-Asher, N. Farber, and S. Levinson, "New Proportional Navigation Law for Ground-to-Air System," Journal of Guidance, Control, and Dynamics, Vol. 26, No. 5, pp. 822-825, DOI: $10.2514 / 2.5118$

[17] M. Weiss and T. Shima, "Optimal Linear-Quadratic Missile Guidance Laws with Penalty on Command Variability," Journal of Guidance, Control, and Dynamics, Vol. 38, No. 2, 2015, pp. 226-237, DOI: 10.2514/1.G000738

[18] Shaferman, V. and Shima, T., "Linear Quadratic Guidance Laws for Imposing a Terminal Intercept Angle," Journal of Guidance, Control and Dynamics, Vol. 31, No. 5, 2008, pp. 1400-1412, DOI: $10.2514 / 6.2008-7302$

[19] Zarchan, P., Tactical and Strategic Missile Guidance, fifth Edition, Washington, DC: AIAA, 2007.

[20] Ryoo, C. K., Cho, H., and Tahk, M. J., "Optimal Guidance Laws with Terminal Impact Angle Constraint," Journal of Guidance, Control, and Dynamics, Vol. 28, No. 4, 2005, pp. 724-732, DOI: $10.2514 / 1.8392$

[21] Lee, C. H., Kim, T. H., and Tahk, M. J., "Interception Angle Control Guidance using Proportional Navigation with Error Feedback," Journal of Guidance, Control, and Dynamics, Vol. 36, No. 5, 2013, pp. 1556-1561, DOI: 10.2514/1.58454 
[22] Ryoo, C. K., Cho, H., and Tahk, M. J., "Time-to-go Weighted Optimal Guidance with Impact Angle Constraints," IEEE Transactions on Control Systems Technology, Vol. 14, No. 3, 2006, pp. 483-492, DOI: 10.1109/TCST.2006.872525

[23] Ohlmeyer, E. J. and Phillips, C. A., "Generalized Vector Explicit Guidance," Journal of Guidance, Control, and Dynamics, Vol. 29, No. 2, 2006, pp. 261-268, DOI: $10.2514 / 1.14956$

[24] Lee, C. H., Kim, T. H., Tahk, M. J., and Whang, I. H., "Polynomial Guidance Laws Considering Terminal Impact Angle and Acceleration Constraints,” IEEE Transactions on Aerospace and Electronic Systems, Vol. 49, No. 1, 2013, pp. 55-73, DOI: 10.1109/TAES.2013.6404092

[25] Y. I. Lee, S. H. Kim, and M. J. Tahk, "Optimality of Linear Time-Varying Guidance for Impact Angle Control," IEEE Transactions on Aerospace and Electronic Systems, Vol. 48, No. 3, 2012, pp. 2802-2817, DOI: 10.1109/TAES.2012.6324662 
2018-04-19

New insights into guidance laws with terminal angle constraints

\author{
Lee, Chang-Hun
}

AIAA

Lee C-H, Seo M-G, New insights into guidance laws with terminal angle constraints, Journal of Guidance and Control, Vol. 41, No. 8 (2018), pp. 1832-1837

http://dx.doi.org/10.2514/1.G002817

Downloaded from Cranfield Library Services E-Repository 